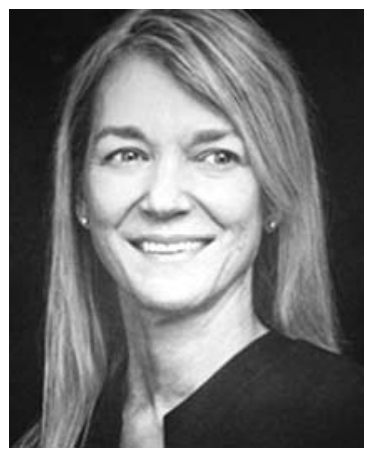

\title{
A Conversation with Cori Bargmann
}

\author{
INTERVIEWER: JAN WITKOWSKI \\ Executive Director of the Banbury Center \\ at Cold Spring Harbor Laboratory
}

Cori Bargmann is Torsten N. Wiesel Professor and Associate Director of the Shelby White and Leon Levy Center for Mind, Brain and Behavior at the Rockefeller University and an Investigator of the Howard Hughes Medical Institute.

Jan Witkowski: Tell us about the system you're interested in.

Dr. Bargmann: My work is with the simplest animal being discussed at this meeting, the nematode worm Caenorhabditis elegans. It has only 302 neurons but is nevertheless a real animal. It moves around, it decides what it likes and doesn't like, it learns from past experience and uses that to affect future decisions. And we can understand those kinds of basic processes using this very simple nervous system, and relate what's going on in an individual's brain to its behavior.

Jan Witkowski: So, what aspect of its behavior do you study?

Dr. Bargmann: Most of my lab studies the response to odors, and that's because odors are the things that worms are most interested in. They are so well specialized for detecting odors that $10 \%$ of their genome encodes $\mathrm{G}$ protein-coupled olfactory receptors. To give you a comparison, humans are thought to be able to smell a trillion different odors, and we do that with about three or four hundred different olfactory receptor proteins. Worms have 2000 olfactory receptor proteins, so we don't even know how many things they can sense. But in any case, this matters to the worm, and because what it smells and what it likes matters to the worm, we study those things to try to gain access to its brain. We have a first-order understanding of the worm's olfactory system. We know what the molecules are that detect odors, we know what neurons those molecules are in, we know which odors the animal finds attractive and why it finds them attractive. So we're now starting to ask the second order of questions, which is why even these simple animals don't act the same every time. When given a particular set of choices, sometimes they'll pick one and sometimes they'll pick the other one. This is true even if you have animals kept under identical circumstances, even if they're getting the identical odor. Even if you have many animals of identical genotypes, you still see this variability. So the question is, where does the brain generate such variability?

Jan Witkowski: So the worms are not robots that always respond in the same way to a given stimulus.

Dr. Bargmann: No, and one of the most striking features of behavior in any animal is that it's variable, that different animals are doing different things. Some of the time they're wandering around, some of the time they seem to be engaged in purposeful action. They're tuning in and out of different behavioral programs.

Jan Witkowski: So what's a worm's response to odor? Presumably, if it's an attractive odor it tends to follow the gradient.

Dr. Bargmann: Yes, that's exactly right. The odors we're studying are attractive odors and one of the responses we look at is whether or not the animal tries to move toward the odor. So, if you take the odor away, for example, it will change its direction. It will look for the odor that is missing, but not always. Sometimes you take the odor away and it doesn't seem to respond at all. It just keeps going on as though it didn't notice. And so, the first question we asked is, well, did it notice? Did its brain even know that the odor was taken away? And since the worm is transparent, we can use fluorescent markers of neural activity in a live animal, without perturbing it at all, to look at the activity of different neurons within the animal's brain. And the answer to the first question is, the worm knows perfectly well that the odor used to be there and now it's not there anymore, because we can see the olfactory neurons generating very strong signals when the odor appears or was removed, and we see the olfactory neurons responding whether or not the animal generates a behavioral response. So, the variability is not because of the ability to detect. The variability is really a choice inside the worm's brain about whether or not to respond to what has just been detected. 
Jan Witkowski: With only 302 neurons, what is the decision process that gives rise to this variable response?

Dr. Bargmann: Since you only have 302 neurons, you have to be able to use the property of those neurons to explain the variability of the behavior. And so we've been working inward, on the one hand, from the olfactory neurons, to look at their activity and the activity of their targets. And on the other hand, we've been working back from the motor neurons that generate the changes in direction, the changes in behavior, and asking: Since these two can be far apart, where do we start to see those two signals separating from each other?

What our results suggest is that whether the animal responds or not depends on what is going on in its brain, its own endogenous brain activity when the signal arrives - the internal patterns of brain activity the animal moves through spontaneously, just as part of its locomotor pattern. If signals arrive at certain times, then they're detected. If signals arrive at other times, they're ignored. And we all know that when your mother's shouting at you, sometimes you respond and sometimes you don't. And essentially that is what the nervous system of the worm is doing. At certain times it's available or accessible to external stimuli and at certain other times it's driven by internal stimuli and will, for just a few seconds, not incorporate external stimuli into its decision-making.

Jan Witkowski: Can you explore what those internal stimuli are?

Dr. Bargmann: There are different forms of internal states of the brain that my lab is interested in. One kind is generated by neuromodulators, molecules that represent certain kinds of motivational and emotional states. So, for example, an animal that is hungry responds very differently to a stimulus than animal that's well fed. Those kinds of differences are to a large degree represented in the neurochemistry of different kinds of neuromodulators operating on certain synapses to make them more or less sensitive. But in the case of this very specific decision, the level at which we're looking is faster than the neuromodulatory systems, and we think it's transient network state. The animal will respond on minute one, respond on minute two, not respond on minute three, respond again on minute four. We think that those variable responses are reflecting shifts in the activity state of the internal circuits. What people have seen for many years in more complex brains, and we are now seeing in worm brains as well, is that different neurons often have collective patterns of activity rather than completely independent activity. One of the terms used to describe this, based on work from John Hopfield and David Tank in the 1980s, is an "attractor state," when groups of neurons tend to become coactive, then maintain each other's activity. So a group of neurons will seize control of the nervous system and maintain that control through their activity. These sorts of activity patterns are what we think allow animals to be responsive or nonresponsive, when temporary patterns of activity representing groups of neurons with collective activities are sensitive or insensitive to other kinds of input. Our work points in that direction. We see that for a smallish number of neurons. Work from other groups, Alipasha Vaziri and Manuel Zimmer's labs, has allowed them to look at the activity of the entire worm brain simultaneously. And they see strong evidence for large groups of neurons that become coactive and then become inactive, perhaps remaining active or in an active state for tens of seconds before flipping down into an inactive state.

Jan Witkowski: What's the functional significance of these states?

Dr. Bargmann: I think that's a question we need to explore. But one influence on us is the idea that an given time, you want to select one action and suppress other actions. In any given circumstance, the worst thing you can do is essentially seize up and try to do three things at once. So perhaps the nervous system commits to particular kinds of actions, suppresses alternative actions, and then over some period of time will allow new actions to take place. And this concept of action selection can be useful in a variety of different circumstances.

Jan Witkowski: So, changing the subject, you've been involved with the Brain Initiative Project.

Dr. Bargmann: A year ago President Obama announced that there was going to be a grand challenge in neuroscience to understand the brain, analogous to the space program or the war on cancer, or the human genome project. When that was announced, the National Institutes of Health director Francis Collins decided that, rather than simply jump in, he wanted to have a rigorous scientific planning process. So, he asked a group of 15 external scientists in different areas of neuroscience but also engineering, clinical science, chemistry, physics, and genetics to get together and to ask, what are the important problems that we need to study in the brain? What are the best approaches to take to solving them? How would you do that? How much would it cost? I'm co-chair of that planning committee together with Bill Newsome from Stanford University, and we have spent the past year consulting with ourselves and consulting with many, many other neuroscientists and scientists in general to try to answer those questions.

The first year the NIH committed 40 million dollars to starting this new project. This is nothing to sneeze at. The second year the NIH has said that they will spend $\$ 100$ million on this project, so they like the plan that we came up with in year one enough to double their investment. And we're just about to turn in a final report after a year of planning and we'll see if the NIH continues to show enthusiasm. But I think there's been quite broad support for the idea that we're at a time in neuroscience where we can study brains at a level that we've never been able to study them before. For 50 years we've been able to look at individual neurons and their activity, and for 30 years we've been able to look at whole brains by fMRI, and by imaging. Now it may be possible to look at the networks of neurons and the communicating circuits of neurons that transmit information at high speed, so that 
we can look at the brain both at high resolution and look at the big picture of many neurons at once. There's a sense that this kind of circuit activity is going to be very important in understanding normal brain function, and also that it may provide a new way to think about what to do for neurological, psychiatric, and brain injury disorders. So, there seems to be broad support for the idea that this is the time to tackle this problem.

Jan Witkowski: And so is it a technical project, in the sense of looking at neurons, looking at their physical connections, at the local level and the brain level, analogous to the way the human genome project was a technical setting of a foundation for future developments in human genetics?

Dr. Bargmann: Yes, I think what inspires the idea of the Brain Initiative now is truly remarkable technical advances that have occurred over the past five to ten years. The first has been the ability to record from many neurons at once, hundreds of neurons at once instead of just one. The second has been the development of optogenetics, methods for perturbing neuron activity and not just watching the activity, but actually linking it to causes. And the third has been remarkable advances in computing technology that let you take very large data sets and make sense of them, and also give you theoretical and modeling capability. But, all of those things are still pretty far from what they would need to be to really address how a circuit works. When you're talking about the circuits that are involved in memory formation, or percep- tion, it's not a hundred neurons you might be thinking about, it might be a million neurons. The first stages of thinking about the Brain Initiative are how to make these methods more powerful, more scalable, less expensive, disseminating them broadly, making them available to a large number of people - developing the technology that would make these large-scale views of brain activity much more powerful. For the first few years, that will be the main investment, and then over time, it will shift to using those tools to asking questions, with some of both at both times.

Jan Witkowski: How long will the project last?

Dr. Bargmann: We were initially told as a working group to think about the first year, then after we handed in the report we were told to keep thinking, and I think that there is a general sense in scientific planning that to really accomplish something in science takes more than 1 or 2 years. Real scientific progress is something that tends to take at least 5 years to get going because you need to set things up, you need to be able to take wrong turns and then correct them. And there's also a sense that if you're thinking out more than about 10 years, you're just going to be hopelessly out of date by the time it's done. And so, I think in real terms people have tended to try to think 10 years ahead, where you think in a lot of detail about what's going to happen in the next couple of years, you think in moderate detail about what might be possible in 5 years, and then you just give a very rough sense of what might be possible at the end of that. 


\section{$\$_{\text {CSH }}^{\infty}$ Cold Spring Harbor Symposia SYMPOSIA On Quantitative Biology}

\section{A Conversation with Cori Bargmann}

Cold Spring Harb Symp Quant Biol 2014 79: 260-262

Access the most recent version at doi:10.1101/sqb.2014.79.04

\section{License}

Email Alerting Receive free email alerts when new articles cite this article - sign up in Service the box at the top right corner of the article or click here. 